Revue d'histoire de l'Amérique française

REVUE D.HISTOIRE DE L'AMÉRIQUE FRANÇAISE

\title{
La guerre de Sept ans et la civilisation canadienne
}

\section{Guy Frégault}

Volume 7, numéro 2, septembre 1953

URI : https://id.erudit.org/iderudit/301591ar

DOI : https://doi.org/10.7202/301591ar

Aller au sommaire du numéro

Éditeur(s)

Institut d'histoire de l'Amérique française

ISSN

0035-2357 (imprimé)

1492-1383 (numérique)

Découvrir la revue

Citer cet article

Frégault, G. (1953). La guerre de Sept ans et la civilisation canadienne. Revue d'histoire de l'Amérique française, 7(2), 183-206.

https://doi.org/10.7202/301591ar d'utilisation que vous pouvez consulter en ligne.

https://apropos.erudit.org/fr/usagers/politique-dutilisation/ 


\section{LA GUERRE DE SEPT ANS ET LA CIVILISATION CANADIENNE ${ }^{1}$}

Nous voici en 1754. Il y a dix ans que les forces s'alignent en Amérique et dans le monde en vue d'un conflit qui sera la première guerre mondiale des temps modernes. Depuis 1713, le Canada lutte pour reculer l'échéance inscrite dans le traité d'Utrecht. On se souvient de ce que comporte ce fameux traité: il consacre l'affaiblissement de la France, et l'umoindrissement de la puissance métropolitaine se traduit tout de suite dans la géographie de l'empire français d'Amérique, dont le Canada constitue l'élément vital. Le traité d'Utrecht dépouille la Nouvelle-France de la baie d'Hudson, de Terre-Neuve et de l'Acadie; il installe aussi la Grande-Bretagne au sud du lac Ontario et du lac Erié, point de communication de l'Amérique française: de ce point de communication, les Anglais entendent faire un point de rupture. Durant quarante ans, la Nouvelle-France s'efforce d'éluder les dispositions de l'accord international de 1713. Il existe une expression américaine qui rendrait exactement ma pensée: après 1713 le Canada vit sur du temps emprunté. Voilà que l'échéance arrive en 1754.

En 1754, au cœur de la région disputée de l'Ohio, au confluent des rivières Allegheny et Monongahela, le marquis du Quesne fait construire le fort qui porte son nom. Il le fait bâtir sur les fondations d'un poste que les Virginiens avaient eux-mêmes entrepris d'ériger. La même année, George Washington surprend et tue Jumonville dans la région de l'Ohio. La même année toujours, et toujours dans la même région, Villiers, par représailles, assiège le fort Nécessité, où

1. Nous publions ici l'un des cours professés par le professeur Guy Frégault dans la chaire de civilisation canadienne-française, récemment fondée à l'Université de Montréal. 
s'est enfermé Washington, et il force celui-ci à signer une capitulation déshonorante dans laquelle le vaincu reconnaît avoir assassiné Jumonville. Le 7 juillet 1754, Villiers rentre au fort Du Quesne. S'il n'a pas poussé à fond les avantages que lui a donnés sa victoire, c'est qu'il agit comme si l'on n'était pas en guerre. De fait, la paix n'a pas été officiellement rompue. La Nouvelle-France a le dernier mot; du moins parait-elle l'avoir. Pourtant, les colonies britanniques possèdent une puissance massive, incomparablement plus grande que celle de l'Amérique française. Comment expliquer que les plus faibles se conduisent comme s'ils étaient les plus forts?

C'est que les colonies britanniques sont bien éloignées de présenter un front commun contre le Canada, où l'unité de la direction assure une étonnante efficacité à presque toutes les initiatives du gouvernement local. Le congrès d'Albany permet de constater jusqu'à quel point les colonies anglaises sont désunies. Des délégués de la Pennsylvanie, du Maryland, du New-York, du Connecticut, du New-Hampshire, du Rhode-Island et du Massachusetts se réunissent à Albany (New-York) le 19 juin 1754. Les délibérations se poursuivaient donc pendant que Washington, après avoir battu trop facilement Jumonville, se faisait écraser à son tour. Les nouvelles alarmantes de l'Ohio ne pouvaient pas manquer de faire impression sur les délégués des diverses provinces. Ces derniers se rendaient parfaitement compte qu'une crise grave se développait. Ils comprirent la nécessité d'agir de concert. Le congrès nomma un comité chargé de préparer un projet d'action commune. Benjamin Franklin élabora ce plan. Il cherchait à contenter tout le monde: il prévoyait un conseil fédéral de délégués désignés par toutes les provinces; ce conseil tiendrait une session annuelle au cours de laquelle il exercerait pour le bien de tous les pouvoirs suivants: faire des lois et nommer des fonctionnaires chargés de les appliquer, lever des armées, administrer les affaires indiennes et, naturellement, voter des impôts. Mais les diverses législatures rejetèrent ce beau plan, et le gouvernement impérial l'accueillit avec froideur. Philosophe, Franklin expliquait que son projet respectait trop l'autonomie coloniale pour plaire au ministère et qu'il soutenait trop la prérogative royale pour recevoir l'assentiment des assemblées provinciales.

En conséquence, ainsi que le souligne le professeur L.-H. Gipson, les colonies, tout en considérant la conduite des Franco-Canadiens 
comme une suite d'empiétements odieux sur la frontière acadienne et sur la ligne de l'Ohio, ne prirent que des mesures dérisoires pour défendre leurs propres intérêts. La Virginie, dont Dinwiddie estimait, en 1754 , la population à 230,000 habitants et la milice à 27,000 hommes, la puissante Virginie mit plusieurs mois à recruter 600 hommes. Le Maryland, peuplé par plus de 150,000 habitants, leva un contingent de 100 hommes. La Caroline du Nord engagea pareillement 100 miliciens. La Caroline du Sud se construisit un fort. Les assemblées du New-Jersey et de la Pennsylvanie ne firent rien du tout.

Les colonies voulaient bien voir les Français évacuer l'Ohio, mais aucune d'entre elles ne consentait à les en déloger. C'est alors que le duc de Newcastle, qui dirigeait le gouvernement britannique, déclara (fin juin 1754) qu'il était impératif de ne pas abandonner les colonies, de maintenir les droits de l'Angleterre en Amérique et de contraindre les Français de mettre fin à "leurs tentatives hostiles de déposséder la Grande-Bretagne de ses droits". Les événements prenaient déjà la tournure qu'ils allaient conserver au cours des années à venir: la métropole ferait la guerre des colonies. George II reculait devant la perspective d'expédier des troupes au Nouveau Monde. L'énergique duc de Cumberland fit taire les hésitations du souverain et le fit consentir à l'envoi de deux régiments en Amérique. Le major général Edward Braddock devint commandant en chef des forces armées des colonies; celles-ci reçurent l'ordre de fournir deux régiments pour marcher aux côtés des unités anglaises.

En 1754, ce sont les Américains - ceux de la Nouvelle-France et ceux des colonies britanniques - qui ont commencé à se battre. En 1755, les métropoles, sous l'impulsion de l'Angleterre, entrent dans le conflit derrière leurs colonies. Le véritable sens de la guerre de Sept ans, c'est qu'elle se fera en vue de l'hégémonie mondiale. La Grande-Bretagne ne comprend pas immédiatement tout ce que ce conflit implique. Il ne faut pas prêter aux hommes d'Etat britanniques plus de lucidité qu'ils n'en avaient; il leur suffisait d'ailleurs d'en avoir plus que leurs adversaires, les politiciens français. 
On l'a observé, pendant la guerre de la Succession d'Autriche (1744-48), le gouvernement britannique avait manifesté une indifférence au moins relative à l'égard de l'acquisition de nouvelles colonies. Le même état d'esprit subsiste en 1755. Horace Walpole pouvait écrire: "Les partisans du ministère envoient au diable les colonies [ damn the Plantations] et se demandent si nous allons nous engager pour elles dans une guerre". Les colonies, et non la métropole, éprouvaient le désir des conquêtes. Des préoccupations d'ordre presque exclusivement économique dominaient alors les conceptions impériales des politiques anglais, et, à leurs yeux, le Canada ne prunait pas une grande valeur économique. Pour eux, le Canada ne pouvait rien produire que des fourrures. La Grande-Bretagne, ils le savaient, pouvait avoir des fourrures sans avoir le Canada: après tout, elle en tirait déjà beaucoup de la baie d'Hudson et du NewYork.

La Grande-Bretagne ne souhaitait pas s'emparer du Canada. Elle désirait toutefois soustraire ses colonies à la menace d'encerclement qui compromettait leur développement. Le fort Du Quesne symbolisait ce danger. Du reste, les provinces américaines criaient au secours et ne savaient pas se défendre. Il est important d'observer qu'au moment où Newcastle déclarait nettement sa volonté de ne pas abandonner les colonies puisqu'elles avaient besoin d'aide, le ministre français de la Marine écrivait au gouverneur et à l'intendant de la Nouvelle-France que, si cette dernière continuait à coûter aussi cher, le roi la laisserait tomber. Le cours de l'histoire se précisait.

Menée pour l'hégémonie mondiale, la guerre de Sept ans allait être une guerre coloniale et, par conséquent, maritime, avec un front européen. La France va se laisser entraîner de plus en plus dans la guerre continentale, alors que l'Angleterre accomplit un effort énorme dans la guerre coloniale. Tout en utilisant le plus possible ses alliés en Europe, la Grande-Bretagne expédie de nombreuses troupes régulières en Amérique; la France se dépense sans compter en Europe alors qu'au Nouveau Monde elle laisse retomber le plus lourd du fardeau de la guerre sur les coloniaux. En 1759, devant Québec, les unités régulières de l'Angleterre fourniront le gros des assaillants, et les milices coloniales fournissent le gros des défenseurs de la capitale. 
Guerre coloniale et, par conséquent, maritime: cela va de soi. Entre les pays américains et les métropoles, la circulation emprunte les routes transatlantiques. Qui a la marine la plus forte commande ces communications. Comment la puissance maritime est-elle répartie entre la France et l'Angleterre? Au début des hostilités, celle-ci dispose de 130 vaisseaux de ligne et celle-là, de 67. La France n'est pas seulement inférieure par son armement, elle l'est aussi par sa tactique navale. Le ministère français de la Marine impose à ses commandants d'escadres des opérations essentiellement défensives; il regarde l'arme navale comme une simple auxiliaire des armées de terre. En vue de conserver ses unités, il interdit à ses marins les engagements à forces égales. Les navires français ne doivent jamais attaquer; si la rencontre ne peut s'éviter sans déshonneur, ils ont la consigne de forcer l'adversaire à donner le premier. La formule est celle-ci: "ne pas compromettre les armes du Roy"; elle s'étale dans presque toutes les instructions des commandants. Tactique logiquement conçue pour conserver les navires; en même temps, tactique bien faite pour déprimer le moral de la marine: elle ne manquait pas d'une certaine efficacité, - ce que Mahan n'avait pas compris et ce qu'a bien vu le professeur Graham - mais elle découlait de la faiblesse de la France sur mer et, à la longue, elle contribuait à accentuer son infériorité.

Quand le gouvernement français constata que la Grande-Bretagne se disposait à intervenir en Amérique, il intervint de son côté. Il y envoya un Allemand, le baron Dieskau, avec 3,000 hommes, dont 1,000 destinés à l'Ile Royale et le reste au Canada. Dieskau, Braddock: les deux généraux ne devaient jamais se mesurer.

Braddock était parti avec les instructions suivantes: débarquer ses régiments en Virginie et en lever deux autres dans les colonies, ce qui lui donnerait environ 4,000 hommes; se mettre en campagne au commencement de 1755 et commencer par chasser les FrancoCanadiens des forts qu'ils avaient érigés dans le secteur de l'Ohio; de là pousser à Niagara et en déloger l'adversaire; pendant ce temps, lancer les coloniaux contre Saint-Frédéric, sur le lac Champlain; enfin, détruire le fort Beauséjour, dans l'est, en liaison avec le vicegouverneur de la Nouvelle-Écosse, Lawrence. En somme, la mission de Braddock est d'anéantir tout d'un coup l'œuvre de relèvement que la Nouvelle-France poursuivait depuis 1713 dans l'Ohio, 
sur le lac Ontario, sur le lac Champlain et à la frontière acadienne. Il s'agissait, en une saison, de défaire le travail de quarante ans. L'objectif ne manquait pas d'ampleur; il en avait trop.

Devant cette offensive, quelle est, en 1755, la situation du Canada? Depuis dix ans, le pays n'a pas déposé les armes. Il donne des signes de fatigue. Forcés de tenir les frontières, les Canadiens n'ont pas le loisir de mettre le sol en valeur. Leurs forces baissent et l'agriculture décline. "Les habitants sont épuisés, écrit Vaudreuil (1755); ceux qui depuis plusieurs années ont pris des terres n'ont pu seulement les défricher". Vers le même temps, Bigot corrobore les dires du gouverneur: "Les Canadiens diminuent beaucoup. Il en est mort un grand nombre de fatigues et de maladies". Par suite de la déficience de l'agriculture et des exagérations de l'entourage de l'intendant, qui contrôle le commerce, l'approvisionnement fait défaut. Le 30 octobre 1755, Vaudreuil rapporte qu'il se voit obligé d'importer des denrées de Louisbourg. Bigot lance des ordonnances destinées à économiser les vivres. L'arrivée des bataillons de Dieskau ajoute à la disette et fait monter les prix. On a les prix de 1751 et ceux de 1755: d'une façon générale, ils ont augmenté de $25 \%$ à $40 \%$.

Quand Vaudreuil rentre au Canada en 1755, il y a déjà plus de deux ans qu'il n'a pas vu l'Amérique. En lui remettant le gouvernement, Du Quesne répète avec insistance dans ses lettres qu'il a pris toutes les précautions et que la colonie est prête à tout événement. Le 10 juillet Vaudreuil se plaint auprès de la Cour: "J'arrive dans un pays où l'on m'assure que tout est tranquille, je n'y trouve en conséquence aucun approvisionnement ni de voitures faites et il me faut faire face de tout côté aux entreprises des ennemis sous moins de six semaines". Quinze jours plus tard, il ajoute: "Je n'aurais eu garde de m'y attendre, fondé sur la certitude que M. le marquis Du Quesne me donnait que le gouvernement était tranquille, qu'il avait prévu à tout et qu'il n'y avait pas d'apparence qu'on dût craindre aucun mouvement de la part des Anglais". Ni sur le plan économique, ni sur le plan militaire, le Canada n'était prêt à la guerre. Pouchot affirme dans ses Mémoires qu'il n'y avait ni vivres ni armes dans les magasins du roi, et cela, explique-t-il, visant Du Quesne et Bigot, parce que chacun songeait plus "à son intérêt particulier qu'au bien du service." 
Ne nous attardons pas aux opérations militaires qui se sont déroulées en Amérique entre 1755 et 1760. Retenons cependant qu'elles se divisent en deux périodes: jusqu'en 1757, le Canada connaît surtout des victoires; à partir de 1758, il connaît surtout des revers. A la fin de 1758, Louisbourg est tombé, l'Acadie n'existe plus, la frontière de l'Ohio s'est effritée et celle du lac Ontario est percée. Le cercle se resserre autour de la colonie. Il part alors du Canada trois émissaires, deux envoyés par Montcalm et un dépêché par Vaudreuil, pour mettre la métropole au courant de cette situation désespérée et demander des secours.

En France, la colonisation a ses rares partisans et ses nombreux adversaires. Ces derniers sont à la mode, ce sont les maîtres de l'opinion publique. Leur idée pourrait se résumer dans le mot souvent cité de Voltaire: "J'aime beaucoup mieux la paix que le Canada et je crois que la France peut être heureuse sans Québec". Ce serait toutefois une erreur que de prendre l'opinion des écrivains, si influents soient-ils, pour celle des milieux politiques. La doctrine des politiques se partage entre plusieurs nuances. Arrêtons-nous à deux positions extrêmes.

La première est celle d'un fonctionnaire supérieur de la Marine, le marquis de Capellis. En décembre 1758, on voit Capellis conseiller au ministère dont il relève d'abandonner à l'Angleterre tout ce qui, sur la carte, se situe au sud du Saint-Laurent, en échange de TerreNeuve, de Minorque et de Gibraltar. Il raisonne que "la France n'est pas en état de peupler un pays aussi vaste que le Canada" et que le Canada produit peu à l'Etat en temps de paix, tout en lui coûtant excessivement cher $\epsilon$ temps de guerre; il aurait mieux valu, conclut-il, consacrer tout cet argent à la construction de navires plutôt que de l'engager inutilement dans l'entreprise canadienne.

Quelques semaines plus tard, un ancien collègue de La Galissonière, le marquis de Silhouette, affirme que la France doit conserver le Canada parce qu'un grand Etat ne peut pas se passer de colonies. Il s'oppose à ceux que l'on traiterait aujourd'hui d'isolationistes. Si, écrit-il, leurs vues triomphaient, "on pourrait voir renaitre dans peu le système gothique que la France peut se passer de colonies et qu'elle n'a besoin que de laboureurs et de soldats. La 
Russie ne manque point de soldats, elle ne manque point de laboureurs, puisqu'elle envoie des blés au dehors; la Russie cependant reçoit des subsides des puissances étrangères: tant il est vrai que quelque chose de plus est nécessaire pour la dignité, la grandeur et la puissance d'un Etat."

C'est dans ce conflit d'idées que se décide le sort du Canada. Les grandes lignes de la politique relative à la Nouvelle-France furent discutées et adoptées lors d'une séance du Conseil du roi, tenue le 28 décembre 1758. Un document daté de ce jour en rapporte les délibérations. Le Conseil commence par observer, que pour accorder au Canada les secours nécessaires, il faudrait disposer de toutes les forces navales de la France. Ce serait, réfléchit-on, et cette réflexion est caractéristique, "risquer la marine entière de Sa Majesté sans certitude du succès". Les conseillers ne s'arrêtent pas davantage à une telle perspective.

Que faire alors? On répond que la meilleure solution serait sans doute, comme le conseille Montcalm, de raccourcir les frontières du Canada en évacuant les avant-postes qui lui restent et de masser entre Québec et le lac Ontario les forces réduites que la colonie peut encore opposer à l'envahisseur. Jusqu'ici, la pression britannique s'est exercée sur la périphérie de la Nouvelle-France; ce plan l'amènera à peser sur le cœur du pays, éventualité que Vaudreuil avait toujours voulu éviter par considération pour le bien-être de la population canadienne.

Cependant, poursuivent les conseillers, il restera toujours à craindre que les ennemis ne fassent irruption avec des armées trop supérieures pour qu'il soit possible de leur résister: c'est peut-être donner plus de cohésion aux forces défensives que de les réunir sur le Saint-Laurent, ce n'est pas les multiplier. Dans ces conditions, ne serait-il pas plus prudent d'indiquer à Vaudreuil quand et comment capituler? La réponse est catégorique: "Il ne paraît pas que l'on doive en aucune manière parler à $M$. de Vaudreuil de conditions de capitulation dans un cas forcé de dernière extrémité, car alors la colonie ne pourra que subir les lois du vainqueur."

Ainsi, la consigne sera de se battre jusqu'au bout, puis de se soumettre à la plus vieille "loi" de la guerre: "Vae victis!" Mais pourquoi se battre jusqu'au "cas forcé de dernière extrémité"? Dans l'espoir de vaincre éventuellement les Anglais? Evidemment 
non. L'objectif est de conserver "un pied" au Canada parce que, disent les conseillers, quand il s'agira, un jour ou l'autre, de négocier la paix avec la Grande-Bretagne, le roi se fera plus aisément, peutêtre, rétrocéder le Canada si la France l'occupe encore, ne serait-ce que sur un point. De plus, si à la paix, Louis XV se voyait quand même contraint de céder le Canada, il obtiendrait pour la France de meilleures conditions en abandonnant un pays encore partiellement occupé qu'en livrant un pays entièrement évacué: ce qui veut dire que, dans ce cas, le Canada aurait souffert et combattu jusqu'à "la dernière extrémité" en vue de permettre à la France d'obtenir de l'Angleterre un meilleur sort pour elle-même; en d'autres termes, le Canada prolongera une résistance désespérée pour mettre dans le jeu éventuel des diplomates français un atout qui leur fournira le moyen de sortir le moins mal possible d'une partie perdue. Telle est la politique tracée par le Conseil du roi le 28 décembre 1758.

En conséquence, le 3 février 1759, le ministre de la Marine, Nicolas Berryer, annonçait à Vaudreuil qu'il n'enverrait pas un seul vaisseau de guerre à Québec. Comme la France ne ferait rien, le ministre ordonnait au gouverneur de "faire marcher tous les hommes en état de porter les armes, en laissant aux vieillards, aux femmes et aux enfants le soin de continuer les travaux de la terre". Il lui enjoignait aussi de bien vouloir, "par quelque apparition dans les campagnes, exciter les vieillards et les femmes aux ouvrages pour lesquels les hommes employés à la défense de la colonie ne pourront pas vaquer. Une pareille conduite de votre part vis-à-vis d'un peuple qui vous a été attaché de tout temps ne pourra que produire un très bon effet sur les esprits."

\section{IV}

Voilà ce qui se passait en France. En Angleterre, comme l'établit le professeur Gipson, le tableau est bien différent. Au début de 1758, Pitt avait préparé des plans pour employer 50,000 hommes contre la Nouvelle-France: il en avait destiné 29,000 (9,500 réguliers et 20,000 provinciaux) à une offensive par le lac Champlain, en direction de Montréal; il en avait lancé 6,000 contre le fort Du Quesne et 14,600 sur l'Ile Royale. La chute de Louisbourg et celle du fort 
Du Quesne en avaient résulté. En 1759, la Grande-Bretagne poursuit ses efforts avec détermination.

Nous savons ce qu'avait décidé le conseil de Louis XV à la fin de 1758 et comment le secrétaire d'Etat à la Marine avait exécuté ces décisions. Il est instructif de considérer ce que la Grande-Bretagne accomplit au même moment. De décembre 1758 à mai 1759 , le gouvernement anglais fait voter par le Parlement plus de L12,750,000 (soit près de 320,000,000 de francs) pour soutenir sa politique de guerre. Le 9 décembre 1758, le premier ministre envoie une lettre circulaire aux gouverneurs des colonies américaines, leur demandant de fournir 20,000 hommes pour appuyer les troupes du roi qui envahiront le Canada par le lac Champlain. En retour, la métropole s'engage à rembourser les frais qu'auront entraînés le recrutement, la solde et l'équipement des unités coloniales. En somme, le gouvernement britannique invite les divers gouvernements coloniaux à contribuer à la guerre et à envoyer leurs factures à la métropole. Les colonies vont en profiter pour remettre de bonnes factures et rétablir ainsi leur position financière. Le Connecticut, par exemple, trouve là le moyen d'éteindre sa dette publique.

Il faut convenir que la Grande-Bretagne connaît de beaux jours. Fière de sa suprématie navale, elle s'applique à la conserver: au printemps de 1759 , elle a trente vaisseaux de ligne en chantier. Son commerce est florissant, florissantes aussi ses colonies; elle est riche, elle s'enrichit tous les jours et ses colonies s'en ressentent. A la même époque, le Canada souffre de la famine, il faudrait le nourrir comme un établissement naissant. La Louisiane est dans la misère. Comme les jours du Grand Marquis sont loin! Si les Antilles françaises parviennent à vivoter, c'est que, depuis 1756, le gouvernement français $\mathrm{y}$ a suspendu son privilège exclusif du commerce au profit des puissances neutres.

Dans les premiers mois de 1759 , Bougainville, qui est encore en France, écrit avec admiration qu'en Angleterre "M. Pitt gouverne, et gouverne bien". Et en France? Lu même Bougainville reconnaît que le roi est "nul"; la marquise de Pompadour, "toutepuissante, premier ministre"; quant à Berryer, "point de grandes vues". Il raconte: "M. Berryer qui, de lieutenant de police de Paris, avait été fait ministre de la Marine, ne voulut jamais comprendre que le Canada était la barrière de nos autres colonies". C'est dans 
le même document que Bougainville rapporte la "parabole" de Berryer "que l'on ne cherchait point à sauver les écuries quand le feu était à la, maison."

Cependant que le secrétaire d'Etat prononçait ces paroles intelligentes, il était loisible de comparer la situation militaire du Canada à celle des Britanniques. Un mémoire secret, signé de Vaudreuil, en avait indiqué les points saillants en novembre 1758: "Les huit bataillons [ de troupes de terre] composent à peine 3,500 hommes. On n'en peut mettre en campagne plus de 1,500 des troupes de la marine [ donc, en tout, 5,000 soldats réguliers ]. Et si on lève 8,000 hommes de milice, les campagnes seront désertes... A peine assez de vivres pour le nécessaire pendant huit mois... Les munitions de guerre sont sur le point de nous manquer."

En face, se massent les forces que les Anglo-Américains vont déployer sur les divers secteurs du front, à l'ouverture de la campagne de 1759. Sur l'Ohio, ils font marcher 7,200 hommes. Ils en lancent 2,000 sur Niagara, poste important par sa position, mais secondaire par ses défenses. Dans la trouée du lac Champlain, le commandant en chef, Amherst, va commander 11,000 hommes, dont 6,000 réguliers. Devant Québec, Wolfe va amener 9,000 hommes, presque tous des réguliers, soutenus par une puissante concentration navale: 22 vaisseaux de ligne, 13 frégates et près de 150 navires de transport.

La politique française se décompose. La politique britannique est celle d'une nation déjà victorieuse. Dès les premiers jours de 1759, le sort du Canada est décidé. Décidé aussi, le sort de la civilisation canadienne.

\section{V}

Lorsque commence, en 1744, le conflit qui ne se dénouera qu'en 1763, la haute courbe de l'histoire du Canada vient de se dessiner; la synthèse de sa civilisation vient de s'effectuer. Le Canada n'en reste pas moins une colonie, un pays jeune qui aurait besoin de la direction politique, de la tutelle économique et de l'appui militaire de sa métropole. En 1755, les hostilités se déclenchent sur une grande échelle. La même année, le ministre de la Marine prévient les administrateurs de la Nouvelle-France: "Malgré la protection 
particulière dont le roi a honoré jusqu'à présent cette colonie, ...Sa Majesté serait bientôt obligée de l'abandonner si on ne parvenait pas à en réduire les dépenses." Quatre ans plus tard, Berryer traduira cet avertissement en langage de garçon d'écurie.

Ces singuliers raisonnements seraient inexplicables, même chez des hommes quelconques, s'ils ne procédaient pas d'une conception particulière des colonies et de la colonisation. Cette conception, on la voit s'exprimer brutalement dans la conduite et même dans les déclarations de certains des fonctionnaires que la France envoie au Canada. Ces derniers montrent simplement qu'ils ont des idées analogues à celles de leurs chefs de Versailles, ce qui n'est, après tout, que naturel. Un des subordonnés de Bigot, le gardemagasin Guillaume Estèbe, définissait textuellement une colonie "un pays où l'on ne va que pour faire fortune". Il rattachait cette déclaration à un principe: "Les colonies n'ont d'autre objet que le commerce, ne se peuvent former, soutenir, accroître, devenir utiles que par le commerce, on n'y va, on ne s'y fixe que pour faire le commerce."

Ainsi, partant de la conception mercantiliste de la colonisation - que les administrateurs coloniaux pouvaient retrouver, longuement expliquée, dans chacune des instructions qu'ils recevaient de la Cour - on aboutit à faire d'une colonie un territoire livré à l'exploitation de commerçants métropolitains. Poussant jusqu'au bout cette idée, on va finir par déclarer que, si le commerce est la raison d'être d'une colonie, tous les métropolitains qui y mettent le pied, quelles que soient leurs fonctions, sont autorisés à s'emparer de son négoce. Bigot affirmera sans sourciller que tous peuvent se faire marchands dans une colonie, "les nobles comme les roturiers, les magistrats comme ceux qui ne sont pas revêtus de cette dignité, les chefs de la colonie comme ceux qui leur sont subordonnés". Les principes défectueux qui inspirent à la France d'abandonner le Canada sont les mêmes, au fond, que ceux qui permettent aux fonctionnaires français de le piller avec une assez bonne conscience.

Je parle de Bigot, je parle des fonctionnaires français en général. Il ne s'agit pas d'une généralisation hâtive. A cette constatation, on peut toujours opposer d'honorables exceptions, mais il n'en reste pas moins que, si l'on se donne la peine d'examiner les dessous des administrations qui ont précédé celle du dernier intendant, de- 
puis celles de Frontenac jusqu'à celle de Bégon, en passant à celle des Raudot pour arriver à celle du marquis de Beauharnais, dans laquelle Dupuy découvrait, disait-il, des "mystères d'iniquité", sans oublier celle de Du Quesne, on doit reconnaître que Bigot n'a fait qu'ériger en système, profitant des occasions que la grande guerre lui offrait, les méthodes de prévarication que bien d'autres avaient pratiquées avant lui. Ce qui imprime un cachet sinistre aux déprédations de Bigot, ce sont les circonstances tragiques au milieu desquelles le magistrat les a perpétrées.

Ces circonstances sont celles d'une guerre totale. Pour les Canadiens, qui s'enfoncent dans le remous du premier conflit mondial des temps modernes, il s'agit effectivement d'une guerre totale. Leur patrie lutte pour conserver sa vie. Ce n'est pas son prestige qui est menacé, c'est son existence. L'adversaire ne travaille pas à diminuer le Canada, il cherche à le supprimer. Quand, en 1759-60, les proclamations de Vaudreuil crient aux Canadiens qu'ils doivent combattre pour leur vie, que la vie de leur pays dépend de l'issue de la guerre, le gouverneur ne fait pas de la propagande fanatique, il dit la vérité toute nue. Et l'histoire lui donnera terriblement raison. La Nouvelle-France disparaîtra en 1763. La proclamation royale effacera de la carte le nom même du Canada, et quand ce nom y réapparaîtra, il ne désignera plus du tout la même réalité qu'autrefois: ce sera alors le nom d'une unité politique et économique absolument étrangère à ce qu'était le Canada du régime français. Voilà une première note à laquelle se reconnaît une guerre totale: le conflit met en jeu la vie du pays.

En voici un deuxième caractère: on est moins en présence d'une guerre d'armées de métier que d'une guerre de peuple. Devant la puissance formidable de l'ennemi, les troupes réglées qui défendent la colonie seraient tout de suite écrasées. Il faut armer le peuple. On calcule qu'environ dix non-combattants doivent travailler à l'arrière pour maintenir un soldat en campagne. La population canadienne, qui est de 55,000 habitants en 1755 , peut difficilement s'élever à plus de 60,000 âmes en 1758. En novembre 1758, nous le savons, Vaudreuil estimait que, s'il appelait sous les armes plus de 8,000 miliciens, les côtes deviendraient désertes, ce qui confirme bien qu'en faisant marcher plus de 6,000 hommes, le pays s'exposait à une grave rupture d'équilibre. Voici qu'à l'été de 1759, Vaudreuil joue 
le tout pour le tout. Il convie ses compatriotes à la défense de Québec. La réponse est spontanée. "On ne s'était pas attendu à voir un aussi grand nombre de Canadiens, rapporte Foligné. On n'avait eu l'intention que d'assembler les hommes en état de soutenir les fatigues de la guerre; mais il régnait parmi ce peuple une telle émulation que l'on vit arriver au camp des enfants de douze à treize ans qui ne voulurent jamais profiter des exemptions accordées à leur âge; jamais sujets ne furent plus dignes des bontés de leur souverain soit par leur constance dans le travail, soit par leur patience dans les peines et les misères qui, dans ce pays, ont été extrêmes: ils étaient dans l'armée exposés à toutes les corvées."

Troisième signe d'une guerre totale: elle n'affecte pas seulement la structure militaire de la colonie, elle s'attaque encore à son armature économique. Nous y reviendrons dans un instant. Observons cependant que le Canada ne sera qu'une ruine économique lorsqu'il déposera les armes, en 1760.

Quatrième caractère d'une guerre totale: elle s'accompagne d'énormes atrocités. A cet égard, un déplacement massif de population comme celui qui est connu sous le nom de la déportation des Acadiens est hautement significatif. La déportation des Acadiens apparaît comme autre chose qu'un mouvement de mauvaise humeur passagère de la part d'une poignée de fonctionnaires britanniques. Elle représente une politique. Outre que l'expulsion en masse a été pleinement autorisée par le gouvernement de la Grande-Bretagne, qui a immédiatement accordé des promotions importantes à celui qui en a été le principal animateur, Charles Lawrence, elle s'est prolongée durant sept ans, d'une façon froide, systématique et calculée, de 1755 à 1762; la dernière cargaison d'Acadiens a été jetée dans des transports britanniques en 1762. La déportation durait encore en 1760 au moment de la reddition de Montréal: en capitulant pour toute la Nouvelle-France, le 8 septembre 1760, Vaudreuil s'efforce d'obtenir du vainqueur qu'il n'exerce pas de représailles sur les Acadiens réfugiés au Canada; il ne peut rien gagner. C'est un fait honteux pour l'humanité. Pas plus que les Acadiens, les Canadiens n'ont échappé aux atrocités; ils ont eux-mêmes fait la guerre cruellement; ils ont participé à d'innombrables expéditions que les Anglais décrivaient comme des "scalping parties". D'autre part, avant même de venir au Canada, James Wolfe s'était promis d'écraser ce 
qu'il appelait "la vermine canadienne". Par une proclamation publiée à l'île d'Orléans, le 27 juin 1757, le général ennemi prévient les Canadiens de "s'attendre à souffrir tout ce que la guerre offre de plus cruel, s'il leur est aise de se représenter à quel excès se porte la fureur d'un soldat effréné". Il tiendra parole. Au commencement de septembre 1759, l'officier Joannès écrit que les Anglais ont "ruiné, dévasté, brûlé et ravagé toutes les habitations depuis Québec jusque dans le bas du fleuve". Pontbriand prédit que les paroisses dévastées "ne seront pas rétablies d'ici en plus de vingt ans dans leur ancien état". De fait, comme l'a remarqué J.-E. Roy, des greffes de notaires de la région québécoise indiquent que, six ans après la chute de Québec, des habitants de la campagne environnante vivaient dans des cabanes, n'étant pas encore parvenus à relever les ruines de leurs demeures.

\section{VI}

Voilà donc le Canada abandonné par la France au moment où il vient tout juste de réaliser sa civilisation et de s'engager dans une guerre totale. Le conflit va naturellement briser les cadres matériels de sa civilisation. Ce dégât se traduit dans un désastre économique, précipité par un gouvernement métropolitain qui se dérobe depuis longtemps à ses obligations et par une administration locale - celle de Bigot - qui ne considère pas le pays comme une société qu'on doit administrer, mais comme une affaire à exploiter.

Nous savons qu'à partir de 1748 , le Canada est le théâtre d'une concentration économique que l'on peut qualifier d'horizontale. C'est l'époque de la "Société du Canada", à côté de laquelle se rangent d'autres entreprises particulières. Les organismes de cette nature se multiplient, et leur ensemble constitue une série de monopoles qui absorbent l'un, les fournitures de l'intérieur, un autre, les fournitures des frontières, un autre encore, la traite des fourrures, un autre enfin, les moyens de transport.

Après 1755, apparaît une concentration verticale de l'activité économique. Le résultat en sera le fameux "marché du munitionnaire", qui livre la colonie à un groupe restreint d'entrepreneurs connus généralement sous le nom de "la Grande Société". Fondée au milieu de tractations sordides, la Grande Société devient mâ̂tresse du commerce intérieur, et son contrat - passé à Québec le 26 
octobre 1756 - lui permet de contrôler les importations et les exportations. Joseph Cadet la dirige. Cadet ne serait-il que le prêtenom d'une compagnie trop protégée? Montcalm l'affirme, d'autres aussi, et Cadet lui-même, à la Bastille, en conviendra. Ce groupe privilégié compte des associés silencieux, dissimulés: Péan, Bigot, Martel, peut-être et même probablement le premier commis à la Marine, La Porte; d'autres associés travaillent au grand jour: Corpron, Maurin, Pénissault. Ces derniers détiennent chacun une part de $8 \%$ des profits. Huit pour cent, cela peut paraître mince. Mais, au cours de l'Affaire du Canada, Corpron déclarera n'avoir pris que des profits légitimes et s'être "contenté d'environ un million, tandis qu'aux termes de l'acte qu'il avait passé avec le sieur Cadet, il en pouvait prétendre deux et demi". Corpron était modeste...

Non contente de jouir des avantages excessifs que lui assurait le "marché" du 26 octobre 1756, la Grande Société vola audacieusement l'Etat, ce qui n'était possible que parce que Bigot, intéressé, fermait les yeux. J'ai décrit ailleurs plusieurs des scandales qui éclatent à l'époque. Voici, presque au hasard, celui de la Britannia. La Britannia était un navire anglais dont un corsaire s'empara en 1758. Elle est amenée à Québec. Bigot en fait adjuger la cargaison. Cadet s'en porte acquéreur au prix de 800,000 livres. Il en revend sans tarder une partie à l'Etat pour 812,000 livres. Mais il lui reste encore les deux tiers du chargement: il les écoule à Québec et aux frontières de l'Acadie, pour en retirer plus d'un million. Un million de profits nets dans une seule opération. La prévarication était devenue cynique. Ce n'est là qu'un exumple.

Avec une pareille administration, on peut imaginer ce qui advenait aux finances publiques. Les dépenses de 1756 avaient dépassé onze millions. En 1757 , elles se portèrent à près de vingt millions et à près de vingt-huit millions en 1758; en 1759, elles atteignirent trente millions, pour retomber à un peu plus de vingt millions st demi en 1760. Il fallait que l'Etat augmentât son propre pouvoir d'achat. Il le fit en émettant de la monnaie de papier. L'inflation décrivit une spirale vertigineuse. Il ne circulait plus dans la colonie que de la monnaie fiduciaire. Elle se multipliait dans la mesure où les budgets de Bigot s'hypertrophiaient.

La Cour tâcha d'assainir la situation monétaire du Canada. Mais elle n'avait jamais rien compris au fonctionnement des finances 
coloniales. Ne s'avisa-t-elle pas d'expédier à Québec des tonneaux de monnaie métallique! En 1755, le corps d'armée de Dieskau en apporta pour 1,200,000 francs. Les renforts de Montcalm en eussent jeté autant dans la colonie, en 1756, si les Anglais n'avaient intercepté une partie de ces espèces. C'est un axiome que la mauvaise monnaie chasse la bonne. La bonne monnaie rend la mauvaise encore plus mauvaise. L'or roula dans les cassettes, et le papier-monnaie fit une chute rapide.

Plutôt que d'envoyer de l'argent, la Cour eût mieux fait de procéder à un nettoyage dans le fonctionnarisme et d'honorer avec exactitude les lettres de change délivrées par le trésor canadien. C'étaient deux mesures également difficiles. Le gouvernement français, qui exigeait pourtant de l'héroïsme de la part des Canadiens, adopta une solution facile: il déclara la banqueroute. Il en avait l'habitude. Il l'avait déjà déclarée au Canada en 1714 et en Louisiane en 1744. Un arrêt du Conseil d'Etat en date du 15 octobre 1759 suspendait le paiement des lettres de change des colonies. Or le papier-monnaie du Canada était gagé sur les lettres de change, qui elles-mêmes valaient quelque chose dans la mesure où le trésor royal les honorait. La monnaie circulant au pays tombait à rien.

Quand la nouvelle de la banqueroute arriva à Québec, Murray, gouverneur de la ville depuis la capitulation, répandit une circulaire dans laquelle, en vue de miner le moral des Canadiens qui résistaient encore, il soulignait fortement le sens de cette escroquerie légale et ajoutait que Louis XV, "à ce qu'il nous paraît, semble croire que les malversations de ses Ministres ont bien contribué à la banqueroute qu'il est obligé de faire". L'armature économique de la NouvelleFrance ne peut pas résister davantage aux forces destructrices qui la rongent à l'intérieur et aux pressions de l'extérieur. Elle s'effondre avec fracas. Le pays qui va passer de l'empire français à l'empire britannique en 1763 est déjà une ruine économique.

Pourquoi? Pour deux raisons. D'abord, il n'a plus de finances. Les moyens de production existent encore. Des forges de SaintMaurice, par exemple, il sortira du fer pendant l'Occupation, entre 1760 et 1763 . L'agriculture se rétablira. Le commerce des fourrures reprendra aussi: l'histoire de nombreuses sociétés, dont la plus célèbre est la Compagnie du Nord-Ouest, en est une preuve surabondante. Pourtant, ce rétablissement, aui s'effectuera avec les 
années, pour aboutir à une prospérité que Plessis célébrera avec grandiloquence - ce redressement ne profitera vraiment qu'aux Anglais. Même si la colonie redevient florissante pour ses nouveaux maîtres, son économie reste pour les Canadiens une grande ruine qui n'a jamais été relevée.

Il y a plus. En 1760, le Canada perd l'équipe de financiers et de négociants qui avaient édifié son économie de guerre depuis 1748 . De qui était constituée cette équipe ? De quarante à cinquante personnages dont les noms s'alignent sur la liste des accusés qui ont comparu devant la commission du Châtelet de Paris, entre 1760 et 1763, au cours de l'Affaire du Canada. Voilà, à vrai dire, le bottin de la finance et du commerce du Canada; Bigot, Bréard, Varin, Cadet, Maurin, Corpron, Pénissault, Le Mercier, Boishébert, Noyan, Fayolle, La Barthe, Grasset de Saint-Sauveur, Estèbe, Martel..., ajoutez-en encore et vous les aurez tous. Ces gens-là étaiunt des profiteurs de guerre. Certains d'entre eux ont beaucoup volé et ont été reconnus pour voleurs. Le tribunal condamna Cadet à restituer six millions; Cadet n'en resta pas moins millionnaire. Péan dut rendre 600,000 francs; il continua à mener un train de grand seigneur.

Efforçons-nous de comprendre le rôle de ces grands carnassiers. Ils ont fait rouler la vie économique du pays. Leur activité a répandu des liasses de lettres de change non seulement dans leur propre caisse, mais aussi dans le peuple, puisque tout le monde, par l'intermédiaire du munitionnaire Cadet, était devenu fournisseur de l'Etat. Tout malhonnêtes qu'ils étaient, ils assuraient une direction aux affaires. Qu'ils aient été coupables, nul n'en doute. Mais coupables envers. qui? On sait de reste qu'ils le furent envers le roi; c'est lui qui les fit juger et - dans les cas où il fut possible d'établir des preuves. suffisantes - condamner: Bigot, à l'exil, Cadet, au bannissement temporaire, d'autres, à l'amende. Coupables, ils le furent encore envers le Canada et les Canadiens.

Ils ont gâté la fin de la Nouvelle-France. Ils n'ont pu se hisser au sommet qu'en éliminant l'équipe qui était déjà en place à la fin de l'intendance de Hocquart. Ils se sont élevés en utilisant des méthodes assez semblables à celles de la pègre, grâce à la complicité intéressée de hauts fonctionnaires et de grands commerçants français. Un mémoire rédigé au Canada vers la fin de 1758 contient ce passage révélateur: "Il est difficile que le Canadien ne soit pas ennuyé de s'en- 
tendre reprocher chaque jour les dépenses immenses qu'on fait pour lui conserver la liberté de sa religion et de ses possessions. Mais ce qui le rebute et le décourage, ce sont les fortunes immenses qu'il voit faire sous ses yeux par différents particuliers qui, envoyés de France pour le gouverner, sont beaucoup plus avides de le dépouiller, en tournant à leur profit les fonds destinés à la colonie." On se montrait du doigt ces individus "comme il y en a eu cinquante qui, de toile vêtus en passant en Canada, ont eu 100,000 francs, 100,000 écus deux ou trois ans après."

Découronné par Bigot de son ancienne élite économique à partir de 1748, le Canada perd sa nouvelle bourgeoisie commerçante en 1760 . En dépit du caractère franchement répugnant de cette dernière, le pays n'en faisait pas moins une pєrte grave. Il ne s'en aperçut pas, et c'est compréhensible. Si l'économie canadienne avait été normale au moment où la colonie passait d'un empire à un autre, c'est-à-dire d'une synthèse économico-culturelle à une autre synthèse économicoculturelle, les Canadiens n'iuraient pu faire autrement que de prendre conscience du cataclysme dans lequel leur civilisation était emportée. Ils étaient trop profondément engagés dans les événements pour saisir le sens de ce fait capital. Les arbres les empêchaient de voir la forêt.

Le 27 septembre 1761, un capitaine de milice canadien, Hervieux, écrit à un correspondant français qui plaignait les Canadiens de subir l'occupation anglaise: "Cessez, Monsieur, d'avoir cette compassion pour nous, notre sort est moins malheureux qu'il n'était cidevant." Hervieux poursuivait que, sous les Anglais, le commerce était devenu libre, que les habitants ne se voyaient plus contraints par la force des armes de livrer contre des chiffons de papier leurs vivres à un munitionnaire, qu'il n'existait plus de monopole et que les prix baissaient. "Car vous savez, concluait-il, que ce sont la tyrannie et l'avarice qui ont causé la perte du Canada, notre patrie". Tout porte à le croire, cet état d'esprit prédomine à l'époque de la conquête. La fin du régime français laisse des souvenirs mauvais et tenaces. C'est ce qui umpêche les Canadiens de voir que la défaite ne peut pas être un bienfait et qu'elle ne saurait avoir d'autre résultat que de défaire ce que des générations laborieuses avaient fait.

Période de mauvais souvenirs, la guerre du Canada le sera pour une autre raison. Quelle est la conduite des troupes françaises 
envoyées à la défense de la colonie en 1755 ? A partir de 1756, elles tombent sous le commandement de Montcalm, qui n'aime pas les Canadiens. Dans les dernières années du conflit, elles se comportent comme en pays occupé. En 1759, pour surveiller Bigot, la Cour envoie le commissaire Querdisien. Celui-ci affirme dans un rapport que Bigot est loin d'être le seul coupable au Canada. Il souligne que les soldats français se livrent à "la licence la plus effrénée". Il ajoute: "Jamais dégât ne fut plus déplorable: moissons, bétail, volaille, légumes, plantations de tabac, tout a été ravagé impitoyablement, dans un pays qui éprouvait déjà la plus grande disette. Toutes représentations faites [ à Montcalm ] ont été inutiles". Le témoignage de Querdisien n'est pas unique. Bigot rapporte aussi que la soldatesque française "s'est attribuée de tuer bœufs, vaches, cochons et volailles et de prendre les pois et autres légumes. Tout le voisinage à présent, à deux lieues à la ronde, est ravagé". Montbeillard écrit de son côté: "Rien n'égale les dégâts commis par les troupes dans les campagnes où l'armée a campé: on s'en plaignait, M. de Montcalm répondait que tout appartenait au soldat, qui, instruit de ses dispositions, se répandait dans les habitations à deux et trois lieues à la ronde". Quand Lévis se voit forcé de lever le siège de Québec, à la mi-mai 1760, un officier note que les soldats se sont tous rembarqués ivres et que leur "maraude" devenait "affreuse". Les Anglais n'ignoraient rien de tout cela. Dans sa proclamation du 27 juin 1759 , Wolfe exploite aux fins de la propagande britannique l'attitude de la France et des Français à l'égard des Canadiens: "La France, incapable de soutenir ses peuples, abandonne leur cause dans le moment le plus critique; et si, pendant la guerre, elle leur a envoyé des troupes, à quoi leur ont-elles servi? A leur faire sentir avec plus d'amertume le poids d'une main qui les opprime au lieu de les secourir". Ce jugement est accablant.

Après le cauchemar, on conçoit que les Canadiens n'aient pas regardé la défaite comme un si grand malheur. Il faut encore se rappeler autre chose. A la fin de 1758, lorsqu'elle abandonne le Canada, la métropole subordonne Vaudreuil à Montcalm. Le vaniteux général se rengorge: il suffit de lire ses lettres à Bourlamaque pour constater jusqu'à quel point il est excité. Un coup d'Etat n'eât pas modifié plus brutalement ce que l'on appelle alors "la constitution de la colonie." Vaudreuil représentait le niveau de vie poli- 
tique que les Canadiens avaient atteint. Montcalm, qui affecte de parler au nom des Français, symbolise un colonialisme rétrograde, même pour l'époque. M. Claude de Bonnault a fortement caractérisé le rôle de Vaudreuil en face de Montcalm: "Avocat, défenseur des Canadiens, Vaudreuil a semblé parfois assumer la responsabilité d'un chef de gouvernement mis en présence d'une armée d'occupation". Le gouverneur et le général ont plaidé l'un contre l'autre devant le gouvernement français. Celui-ci a donné raison à Montcalm et à ce qu'il incarnait contre Vaudreuil et ce qu'il incarnait. Ruine économique, le Canada est aussi une ruine politique quand il passe de l'€mpire français à l'empire britannique.

\section{VII}

Ce qui rendait ce fait tragique, c'est que le Canada avait encore besoin de la France. Rien n'illustre mieux ce point qu'une brève comparaison entre le Canada et les colonies anglaises d'Amérique. En 1775, ces dernières entrent dans leur révolution. En 1783, elles ont gagné leur indépendance. Pourtant, les Etats-Unis d'Amérique sentent qu'ils ont besoin de renouer des rapports économiques avec leur ancienne métropole. Vainqueurs de la guerre de Sept ans, vainqueurs de la guerre de l'Indépendance, ils ne constituent ni une ruine économique ni une ruine politique. Bien au contraire. Cependant leur développement harmonieux exige toujours qu'ils entretiennent commerce avec la Grande-Bretagne; sans abdiquer, naturellement, leur indépendance, sans cesser d'être une nation. A plus forte raison le Canada devrait-il renouer avec la mère-patrie. Mais celle-ci a décidé d'abandonner le Canada. Elle aidera les Etats-Unis à devenir une nation. Elle ne modifie jamais sa décision de laisser tomber le Canada.

Qu'est-ce qui va se passer?

La Grande-Bretagne prend le Canada. Elle disloque la Nouvelle-France. Elle prend le Canada pour elle-même. Elle ne le prend pas pour rendre service aux Canadiens. Dans sa nouvelle colonie, sa fonction normale consiste à faire sa politique. Les "anciens sujets" qui vicnnent s'établir dans la province de Québec - c'est le nom officiel du Canada défait - n'y viennent pas faire la politique des Cancdiens, la vie économique des Canadiens, la vie sociale des Canadiens. Ils viennent y faire leur propre politique, y établir et y renforcer leurs propres cadres économiques, servir les intérêts 
d'une société, la leur, qu'ils forment eux-mêmes et pour eux-mêmes. Ils ne viennent pas refaire la civilisation canadienne, ils ne viennent pas travailler pour les Canadiens. Ils vont faire travailler les Canàdiens pour eux. Le contraire ne serait pas naturel. Le conseil de Louis XV avait bien prévu, le 28 décembre 1758 , ce qui arriverait aux Canadiens en cas de défaite: ils subiraient les lois du vainqueur. C'est ce qui se produit, et il est normal que cela se produise ainsi. Je ne dis pas qu'il est heureux que cela soit arrivé: rien ne pouvait être plus malheureux; mais c'était dans la nature des choses.

Qu'est-ce que le Canada perd donc en 1760 ? Il perd d'abord du territoire. Il se fait enlever la zone du C€ntre-Ouest, vitale pour le développement de son économie et pour le maintien de sa société. Il se fait amputer de ce qu'il n'avait jamais voulu céder depuis 1713, sachant fort bien que laisser aller cette région, c'eût été dégénérer à l'état d'une petite colonie agricole et cesser d'être viable. Le Canada perd ce territoire pour lequel les Anglo-Américains se sont battus contre lui et pour lequel les mêmes Anglo-Américains se battront contre la Grande-Bretagne pendant la guerre de l'Indépendance. Il perd, de plus, ses relations avec la France. Il perd enfin son élite politique et économique. Nous savons que des éléments matériels et des éléments spirituels forment une civilisation. En 1760-63, le Canada voit crouler les éléments matériels de sa civilisation. Il est précipité dans une évolution qui fera de lui un parasite politique collé au flanc d'un organisme étranger, en même temps qu'un parasite économique collé au flanc d'un organisme étranger.

On appelle culture les éléments spirituels d'une civilisation. Les pertes matérielles des Canadiens vont-elles rester sans répercussion sur leur culture? Je voudrais pouvoir répondre oui. Mais répondre oui, comme on l'a fait si souvent, répondre oui avec confiance ne changerait rien aux faits. Je ne dis pas qu'il ne serait pas heureux de changer les faits: rien ne serait plus heureux. Mais une négation passionnée et même passionnément honnête n'a jamais aboli un fait.

Qu'est devenue la culture du Canada ? Cent ans après la défaite, une renaissance littéraire s'opère à Québec. C'est l'école de 1860. Quel en est le caractère? Celui d'une école romantique française. Cette floraison de poèmes et d'essais ne donne que des fleurs de papier. Trente-cinq ans après, l'école de Québec est épuisée, discréditée. L'école littéraire de Montréal lui succède, et sa tendance 
principale est celle du Parnasse français, depuis longtemps déjà dépassé en France. Presque en même temps, se dessine le régionalisme paysan, qui fait fortune, au Canada, à l'époque même où se déclenche le mouvement de l'urbanisation. Rien de plus équivoque que ce régionalisme littéraire. Deux idées complémentaires se répandent: la première veut que le Canadien ait une grande patrie, le Canada britannique, le Dominion of Canada, et une "petite patrie", la paysanne province de Québec, dont la population vit de plus en plus dans les villes; la seconde veut que le Canadien ait une grande littérature, la littérature française, dont il est fier comme s'il l'avait inventée, et une petite littérature régionale, la littérature "canadienne-française", qu'il range dans le même coin que le rouet, le ber et la ceinture fléchée. Les Canadiens parlent de leur culture française, de leur enseignement français, de leur vie française, de leurs institutions françaises. Ils se passionnent pour les querelles littéraires et politiques de la France. Ils sont pour ou contre Napoléon III (Fréchette injurie superbement Bazaine dans ses vers), pour ou contre la République, pour ou contre Mgr Gaume, pour ou contre l'Action française (celle de Paris), pour ou contre Laval (Pierre), pour ou contre Pétain. Ils accueillen, avec respect et attendrissement, comme des maîtres, des agents culturels qui ne sont pas des maîtres, mais des diplomates. Il y a quelques années, un collège de la région de Montréal donnait à ses élèves de nombreux cours de littérature française; il leur offrait aussi quelques cours consacrés aux littératures étrangères; parmi ces dernières, vers la fin de l'année scolaire, il faisait une juste place à la littérature "canadienne-française". Le fait est authentique. Je pourrais en citer d'autres, tout aussi révélateurs, et que je connais d'expérience. Les Canadiens se considèrent comme habitant une petite province littéraire de la France.

Poursuivons la comparaison que j'esquissais il y a un instant avec les Etats-Unis. Les Etats-Unis gagnent leur indépendance. Ils prennent les moyens normaux d'assurer l'existence matérielle de leur civilisation. En même temps, ils rendent possible l'avancement de leur culture. Tout se tient. Les Etats-Unis ne sont pas une province littéraire de l'Angleterre. Les Américains aiment et goûtent Shakespeare comme les Canadiens aiment et gouttent Molière. Ils ont leurs propres savants, leurs propres artistes et leurs propres écrivains. 
Ils fournissent une contribution très importante aux sciences de l'homme et de la nature. Leurs livres sont lus partout, en anglais et en traduction. Dans leurs institutions d'enseignement secondaire et d'enseignement supérieur, la littérature américaine ne passe pas pour une littérature étrangère. Les Américains ne parlent ni de leur culture anglaise, ni de leur enseignement anglais, ni de leur vie anglaise, ni de leurs institutions anglaises. Les Etats-Unis sont un pays qui a réussi; ils ont vraiment évolué de l'état de colonies à l'état de nation.

M. Guy Sylvestre a mené récemment une enquête auprès de nos libraires sur ce que lisent les Canadiens. Il constate: "Les livres canadiens ne représentent qu'une petite partie du commerce des libraires au Canada français. Au plus 15 pour cent". Sans être un fanatique de l'indigénisme, on doit convenir qu'un peuple qui prend ailleurs $85 \%$ de ses nourritures intellectuelles est un peuple qui a une vie intellectuelle de parasite. Encore une fois, je souhaiterais bien pouvoir constater quelque chose de différent. Mais le fait est là.

Posons de nouveau la question: la perte que les Canadiens ont subie, en 1760-63, des éléments matériels de leur civilisation allaitelle rester sans répercussion sur leur culture ? La réponse est non. Les Canadiens restent catholiques. La conquête et la défaite ne les ont pas conduits à l'apostasie. La religion d'un peuple exerce une influence profonde sur sa civilisation. La civilisation d'un peuple vraiment chrétien ne peut pas être la même que celle d'un peuple qui ne l'est pas ou qui a cessé de l'être. Tous le reconnaissent. C'est évident. Mais on peut être chrétien sans être canadien; on peut être catholique sans être canadien: les catholiques étrangers le savent.

Nous venons d'étudier une défaite. Nous avons tâché d'en déterminer les principales conséquences. Le professeur Lower, comme M. le chanoine Groulx le rappelait encore il y a quelques mois, a écrit que la défaite est une espèce d'esclavage. C'est une façon de parler. En réalité, défaite signifie désintégration. Une armée est défaite; après la défaite, il reste encore des soldats, il ne reste plus d'armée. En 1760-63, le Canada est défait; il reste encore des Canadiens, il ne reste plus de Canada. Les vaincus vont-ils reconstituer leur civilisation ? Là réponse appartient à l'historien du régime britannique. 